\title{
Corela
}

Cognition, représentation, langage

16-1 | 2018

Vol. $16, \mathrm{n}^{\circ} 1$

\section{Tradition et changement phonétique dans une variété de contact : l'anglais de Lewis et Harris}

\section{Stephan WILHELM}

\section{(2) OpenEdition}

\section{Journals}

Édition électronique

URL : http://journals.openedition.org/corela/6623

DOI : $10.4000 /$ corela.6623

ISSN : 1638-573X

Éditeur

Cercle linguistique du Centre et de I'Ouest - CerLICO

Référence électronique

Stephan WILHELM, «Tradition et changement phonétique dans une variété de contact : I'anglais de Lewis et Harris », Corela [En ligne], 16-1 | 2018, mis en ligne le 28 août 2018, consulté le 10 décembre 2020. URL : http://journals.openedition.org/corela/6623 ; DOI : https://doi.org/10.4000/corela.6623

Ce document a été généré automatiquement le 10 décembre 2020.

\section{(c) (i) (2)(2)}

Corela - cognition, représentation, langage est mis à disposition selon les termes de la licence Creative Commons Attribution - Pas d'Utilisation Commerciale - Partage dans les Mêmes Conditions 4.0 International. 


\title{
Tradition et changement phonétique dans une variété de contact : l'anglais de Lewis et Harris
}

\author{
Stephan Wilhelm
}

\section{Introduction}

1 La langue anglaise a été introduite assez tard dans les Iles Hébrides, où l'on assiste aux premières tentatives d'éradiquer officiellement le gaélique au XVII siècle. L'organisation anglicane visant à promouvoir l'enseignement de la doctrine chrétienne (Society for the Propagation of Christian Knowledge ou SPCK) joue initialement un rôle notoire dans cette entreprise ${ }^{1}$, mais celle-ci n'est guère couronnée de succès avant la première moitié du XIX e siècle (MacDonald, 1978 : 143; Shuken, $1984: 152$ ). Ce n'est qu'à partir de la deuxième moitié du XIX $\mathrm{X}^{\mathrm{e}}$ siècle, notamment après la promulgation de l'Education Act de 1872, qui bannit le gaélique de la sphère éducative, que l'anglais commence à s'enraciner profondément dans la communauté hébridéenne (MacDonald, 1978:145-158; Shuken, 1984 : 153 ; Robertson, $2011: 27$ ).

2 La variété d'anglais implantée dans les Hébrides est essentiellement le Standard Scottish English (SSE), mais les instituteurs chargés de l'enseigner avaient le gaélique pour langue maternelle. Leur propre anglais était par conséquent fortement influencé par celui-ci sur le plan de la prononciation (Shuken, $1984: 153$ ). Ceci explique en partie les spécificités phonétiques et phonologiques de l'anglais de Lewis et Harris, qui partage certains traits avec le SSE, mais doit aussi ses caractéristiques traditionnelles à l'interférence du gaélique. Le corollaire inverse est vrai: le déclin actuel du gaélique, conjugué à l'accroissement de la mobilité géographique, n'est pas sans conséquence sur cette variété de contact. Ce sont ces deux phénomènes que nous mettrons ici en lumière dans une perspective à la fois synchronique et diachronique. 


\section{Objectifs et données étudiées}

\subsection{Objectifs}

3 En nous appuyant sur des données originales, nous nous proposons tout d'abord d'identifier et de présenter les caractéristiques phonétiques et phonologiques les plus saillantes de l'anglais de Lewis et Harris. Nous mettrons ensuite en lumière plusieurs éléments qui signalent des changements linguistiques en cours. À partir de ces observations, nous réfléchirons enfin à des questions phonétiques et sociolinguistiques plus générales sur lesquelles l'examen de l'anglais de Lewis et Harris apporte un éclairage singulier.

\subsection{Données étudiées}

Les données sur lesquelles nous nous baserons sont :

Environ 1h10' d'enregistrements de deux locuteurs de gaélique, mari et femme, amis de l'auteur depuis plus de quinze années et âgés respectivement de 73 et 72 ans. Ces informateurs ont tous deux grandi dans des zones rurales de Lewis mais vivent à la périphérie de Stornoway depuis 2002. Ces enregistrements comportent des conversations en langue anglaise spontanées et informelles, la lecture d'un extrait de roman contemporain comportant des éléments de dialogue et la lecture d'une liste de mots. Ils ont été réalisés à l'aide d'un appareil enregistreur de marque SONY ${ }^{\circledast}$ (modèle MZ-RH1) et d'un microphone « table » multidirectionnel (modèle ECM-F8).

6 Ces données sont complétées par environ 3h d'enregistrements réalisés à Stornoway et dans diverses zones rurales de Lewis et Harris en 2005 dans le cadre d'un autre projet de recherche (Wilhelm, 2005). Il s'agit essentiellement d'entretiens spontanés avec l'auteur et de la lecture d'une liste de mots.

7 Dans le cadre de ce projet, vingt-deux informateurs résidant à Stornoway avaient été interviewés et enregistrés à l'aide d'un appareil de type Sony TCM-20 DV. Ces informateurs avaient tous grandi à Stornoway, bien que plusieurs aient habité quelques années en Écosse continentale. Ils étaient initialement répartis en trois tranches d'âge pour permettre une observation des évolutions de la langue en temps apparent ${ }^{2}: 1$ ) de 65 à 80 ans ;2) de 35 à 45 ans ; 3) de 17 à 25 ans. Les interviews duraient entre vingt et quarante minutes. Après quelques moments d'échange avec l'auteur destinées à permettre l'établissement d'une atmosphère informelle, les enregistrements visaient à conserver la trace de conversations spontanées dont la durée était comprise entre cinq et quinze minutes et de la lecture enregistrée d'une liste de mots (fournie en appendice). Les informateurs étaient conscients de la visée scientifique de l'entretien et savaient qu'ils étaient enregistrés. Les conversations étaient orientées selon les intérêts qu'ils manifestaient au cours de l'entretien.

8 La liste de mots comprenait les termes sélectionnés par Chambers \& Trudgill (1996: xi) pour révéler la présence de certains contrastes vocaliques (voir les items 1 à 52 dans l'inventaire en appendice). Elle était augmentée d'items destinés à mettre en évidence d'éventuels phénomènes attribués à l'influence du gaélique, tels que la préaspiration des consonnes plosives sourdes (voir section 2.2.1.), le dévoisement des plosives et sibilantes sonores (voir sections 2.2.2. et 2.2.3.) ou la présence de schwas épenthétiques à l'intérieur 
de certaines suites consonantiques (voir section 2.2.9.). Quatre suites de type adverbe + adjectif et trois courtes phrases y avaient également été incorporées pour déterminer si certaines innovations telles que la réalisation glottale de $/ \mathrm{t} /$, la vocalisation de $/ \mathrm{l} /$ et l'antériorisation de $/ \theta /$ et de $/ ð /$ apparaissaient même dans la lecture.

Dans la mesure où de nombreux informateurs affirmaient que l'accent des adolescents de Lewis présentait un caractère nettement distinctif, la décision avait été prise en cours de projet d'interviewer un quatrième groupe d'informateurs âgés de 14 à 17 ans. Des échanges impromptus avec des adolescents avaient ainsi été enregistrés dans les rues de Stornoway ${ }^{3}$. Deux des locuteurs interviewés avaient également consenti à lire la liste de mots. Ces éléments avaient été complétés par des notes recueillies à l'occasion d'échanges informels avec des habitants de Stornoway ou d'observations rapides et anonymes (cf. Labov, 1966).

10 L'ensemble de ces données a été complété et ré-analysé à la lumière d'observations informelles, mais systématiques réalisées par l'auteur durant ses séjours à Lewis et Harris au cours des seize dernières années.

\section{Description des spécificités phonétiques et phonologiques traditionnelles de l'anglais de Lewis et Harris}

\subsection{Traits partagés avec le SSE (Standard Scottish English) et la plupart des variétés d'anglais écossais}

11 Les variétés d'anglais parlées à Lewis et à Harris partagent un certain nombre de traits avec le SSE et la plupart des variétés d'anglais écossais.

\subsubsection{Voyelles}

12 Pour décrire les voyelles de l'anglais de Lewis et Harris, nous utiliserons les ensembles lexicaux proposés par Wells, représentés par des mots-clefs (Wells, 1982 : xviii). Ceux-ci ont été choisis de manière à représenter de façon non équivoque les éléments du système vocalique des diverses variétés d'anglais, indépendamment de l'accent de référence ${ }^{4}$. KIT représente ainsi la voyelle utilisée dans des mots tels que ship, sick, bridge, TRAP la voyelle utilisée dans des mots comme bat, lack, rattle, FACE la voyelle utilisée dans des mots comme tape, raid, steak, etc. Cette approche est adoptée pour éviter de choisir arbitrairement les composantes du système phonémique d'un accent particulier pour servir de base de comparaison.

13 FACE et GOAT: Les voyelles mi-fermées sont invariablement des monophtongues. I n'existe aucun contraste entre de mots tels que wait/weight, ou nose/knows comme c'est le cas dans diverses variétés du nord de l'Angleterre. Dans l'anglais de Lewis et Harris, FACE et GOAT sont généralement plus fermées que leurs équivalents des accents du nord de l'Angleterre.

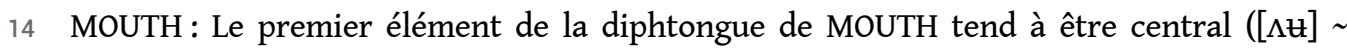
[əu]) (voir aussi Wells, $1982: 406)$. Les éléments les plus fermés et centraux ([əu]) sont observés principalement dans la production des locuteurs les plus âgés. Certains locuteurs les associent surtout au parler de Stornoway. 

fricative vélaire $/ \mathrm{x} /$ dans les mots d'origine gaélique tels que loch ainsi que dans certains toponymes et patronymes (Murdoch, etc.). Ceci corrobore à nouveau les remarques de Shuken (1984: 159).

Fricative labiovélaire : Une distinction existe entre /w/, approximante labiovélaire, et la spirante labiovélaire sourde $/ \mathrm{M} /$. Ces consonnes sont respectivement observées dans des mots comme witch (orthographiés sans $<\mathrm{h}>$ ) et which (qui comportent un $<\mathrm{h}>$ dans leur graphie). La spirante labiovélaire est souvent fortement aspirée, notamment par les locuteurs les plus âgés, au point de s'apparenter d'avantage à une fricative, et sa composante vélaire peut être réalisée de manière très marquée, parfois de manière isolée, à l'initiale du phonème $([\widehat{\mathrm{xw}}]$ ou $[\mathrm{xM}])$. Certains mots qui ne comportent pas la graphie $<$ wh> sont réalisés avec $/ M /$ (par exemple, quite $=>$ [kMaIt $]$ ).

\subsection{Traits caractéristiques des variétés de Lewis \& Harris}

Après avoir recensé les traits que l'anglais de Lewis et Harris partage avec le SSE et autres variétés d'anglais écossais, nous nous pencherons à présent sur ses spécificités phonétiques et phonologiques distinctives. Pour la plupart, celles-ci doivent être attribuées à l'influence du gaélique et, pour cette raison, sont parfois observées également dans certaines variétés d'anglais des Highlands (Shuken, 1984 : 160). 


\subsubsection{La préaspiration des plosives sourdes} préaspiration des consonnes sourdes $/ \mathrm{p} /, / \mathrm{t} /, / \mathrm{k} /$. Cette particularité, singulièrement marquée après les voyelles ouvertes et semi-ouvertes, est essentiellement observable dans la production des locuteurs les plus âgés et/ou vivant en zone rurale, notamment Carloway et Harris. Pat, cat, better, batter sont ainsi réalisés [ $\left.p^{\mathrm{h}} \mathrm{a}^{\mathrm{h}} \mathrm{t}\right],\left[\mathrm{k}^{\mathrm{h}} \mathrm{a}^{\mathrm{h}} \mathrm{t}\right],\left[\mathrm{b} \varepsilon^{\mathrm{h}} t \partial \Gamma_{0}\right],\left[\mathrm{b}^{\mathrm{h}} \mathrm{a}^{\mathrm{h}}\right.$ tər̊]. On observe également un dévoisement des consonnes sonores avant ces plosives ( milk $\rightarrow$ [millk]). La latérale est alors dévoisée, ce qui constitue une forme de préaspiration ${ }^{6}$ . Ce trait caractéristique doit lui aussi être attribué à l'influence des variétés de gaélique de Lewis et Harris. Elle présente d'autant plus d'intérêt que la préaspiration des plosives sourdes, inconnue dans les autres langues celtiques, est observée dans un nombre restreint de dialectes du gaélique d'Écosse et dans certaines variétés de langues nordiques. Il est tentant de penser que sa présence dans certaines régions du nord de l'Écosse résulte d'une influence scandinave.

\subsubsection{Le dévoisement des plosives sonores}

Ce phénomène, considéré par Wells (1982:413) comme caractéristique des localités fortement marquées par l'influence du gaélique, est régulièrement observé dans les zones rurales de Lewis et Harris et dans la production des locuteurs les plus âgés. Il existe alors parfois un risque de confusion entre bet et pet $\left(\left[b \varepsilon_{-}^{\mathrm{h}} \mathrm{t}\right] /\left[\mathrm{p} \varepsilon_{-}^{\mathrm{h}} \mathrm{t}\right]\right)$ ou entre bat et pat ([bo $\left.\underline{a}^{\mathrm{h}} \mathrm{t}\right] /$ $\left.\left[\underline{p a}^{\mathrm{t}} \mathrm{t}\right]\right)$.

\subsubsection{Le dévoisement des sibilantes sonores}

A l'intérieur des mots et en position finale, /z/ et /3/ sont souvent totalement dévoisées (voir aussi Shuken, 1984:157). Cows, rouge, enjoying, years, there's, pleasure sont ainsi

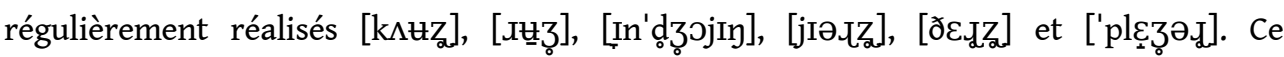
phénomène doit lui aussi être attribué à l'influence du gaélique écossais, dans lequel aucune opposition de voisement n'existe à l'intérieur de la classe des sibilantes (Shuken, $1984: 158)$.

\subsubsection{La palatalisation de / $\mathrm{s} /$ avant $/ \mathrm{t} /$ avant les voyelles antérieures fermées}

Dans l'anglais de Lewis et Harris, /s/ est ordinairement palatalisée dans la suite /st/ avant une voyelle ou une semi-voyelle antérieure fermée (stew $=>$ [ [tju] ; student $=>$ ['Stjudnt $]$ ). Ce phénomène résulte de la transposition d'une règle qui s'applique en gaélique (Shuken, 1984 : 159).

\subsection{5. $\mathrm{TH} / \mathrm{DH}$}

Dans la production des locuteurs les plus âgés, il arrive parfois que $/ \theta /$ and $/ ð /$ soient réalisées comme des fricatives alvéolaires : [s] (something $\Rightarrow>$ ['s $\Lambda$ msin] $]$ ) et [z] (that $\Rightarrow>$ [zat]) (voir aussi Wells, $1982:$ :13). /ð/ est aussi occasionnellement réalisée comme une plosive dentale : [d] $]$ (that $=>$ [dat]). 


\subsection{6. $/ \mathrm{r} /$}

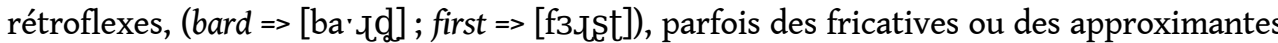

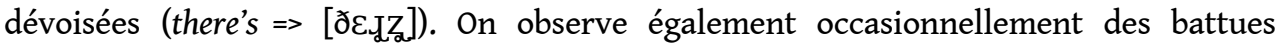
rétroflexes avant les consonnes alvéolaires (choirs $=>$ [kwaĨə[s]). Avant les labiales ou les labiodentales, $/ \mathrm{r} /$ est habituellement réalisé par une battue alvéolaire/post-alvéolaire. Des approximantes post-alvéolaires/rétroflexes sont souvent employées avant $/ \mathrm{g} /$ alors que des battues alvéolaires/post-alvéolaires sont fréquentes avant $/ \mathrm{k} /$ et $/ \theta /$ (worth $=>$

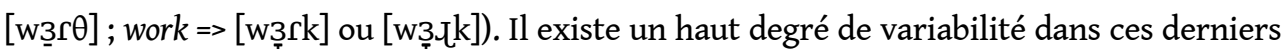
environnements, et il n'est guère aisé d'expliquer les raisons qui président à tel ou tel choix. Ainsi le nom du village du Sutherland Lairg est normalement prononcé avec une approximante rétroflexe directement suivie d'une plosive vélaire alors que le mot gaélique lorg («trace ») l'est d'ordinaire avec une battue alvéolaire suivie d'une voyelle épenthétique.

\subsubsection{La rétroflexion des consonnes alvéolaires} anglais de Lewis et Harris. Shuken $(1984: 156)$ souligne que plusieurs de se informateurs tentaient de l'éliminer de crainte d'être exposés aux moqueries de locuteurs étrangers à la communauté. Nous n'avons observé aucune tentative de ce type, du moins chez les locuteurs les plus âgés. rétroflexes de $/ \mathrm{t} /, / \mathrm{d} /, / \mathrm{n} /$ et $/ \mathrm{l} /$ sont aussi observées systématiquement après $/ \mathrm{r} /$ et parfois après d'autres consonnes ou des voyelles (pot et heed peuvent par exemple être 
respectivement réalisés [pot] et [hi·d]). Ces formes sont cependant moins saillantes que $[\mathrm{s}]$ et $[\mathrm{z}]^{8}$.

\subsubsection{L'usage de variantes claires de /I/ dans tous les environnements}

37 Dans la production des locuteurs les plus âgés, /l/ est souvent clair dans tous les environnements linguistiques. Cette observation s'applique aux données recueillies à Stornoway comme aux observations réalisées dans les zones rurales. Elle corrobore les descriptions que Wells $(1982: 143)$ fournit de l'anglais hébridéen. Une fois de plus, l'origine de cette spécificité est probablement celtique. Le gaélique comporte bien une variante vélaire, voire pharyngée, de la latérale alvéolaire /1/, mais pour ses locuteurs natifs, celle-ci est vraisemblablement si différente de l'approximante latérale de l'anglais qu'elle n'exerce pas d'influence sur leur production dans cette langue9.

\subsubsection{Le schwa épenthétique}

38 Ce phénomène, connu sous l'appellation de svarabhakti, est quasi-systématiquement observé dans les suites consonantiques de type $/ \mathrm{r} /$ ou $/ \mathrm{l} / \mathrm{+} / \mathrm{m} /$ (par exemple, dans farm, film, etc.) et plus irrégulièrement dans celles de type $/ t /+/ r /$ (petrol). Il est vraisemblable que cette spécificité doive être attribuée à l'influence du gaélique, dans lequel le phénomène existe, comme dans toutes les langues celtes (Clement, 1984 : 321). Dans le gaélique hébridéen, l'épenthèse est plus marquée qu'en anglais, la voyelle observée dans les suites consonantiques étant une réplique exacte de celle qui apparaît dans la première

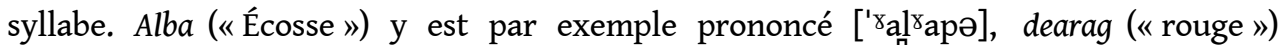
['d'arag], et gorm («bleu ») ['gorom] (Clement, $1984: 321$; Black, $1992: 133)^{10}$.

\section{3. Éléments suprasegmentaux}

39 Pour rendre compte des spécificités phonétiques et phonologiques de l'anglais de Lewis et Harris, il faut aussi prendre en compte des traits prosodiques (ou suprasegmentaux) tels que l'intonation et la qualité de voix.

\subsubsection{L'intonation}

Il n'est pas question de fournir ici une description détaillée de l'intonation de l'anglais de Lewis et Harris, mais nous en décrirons les traits les plus remarquables.

41 À l'instar de certaines variétés associées à des zones géographiques comme l'Irlande du Nord ou Glasgow (Cruttenden, 1994 :155-158), l'anglais de Lewis et Harris possède un système intonatif radicalement différent de celui du RP et du SSE. En l'occurrence, l'une des spécificités de ce système réside en la nature du ton nucléaire employé par défaut en fin d'énoncé déclaratif, parfois interrogatif. Il ne s'agit pas d'une chute simple, mais d'un ton de type rise-fall souvent accompagné d'un allongement de la syllabe pivot (fig. 1).

En fin d'énoncé déclaratif, on note également l'emploi occasionnel de tons de type riseslump, dans lesquels la chute mélodique s'arrête avant le point bas de la tessiture du locuteur (fig. 2). 
Fig. 1 : Ton de type rise-fall employé par défaut dans l'anglais de Lewis et Harris

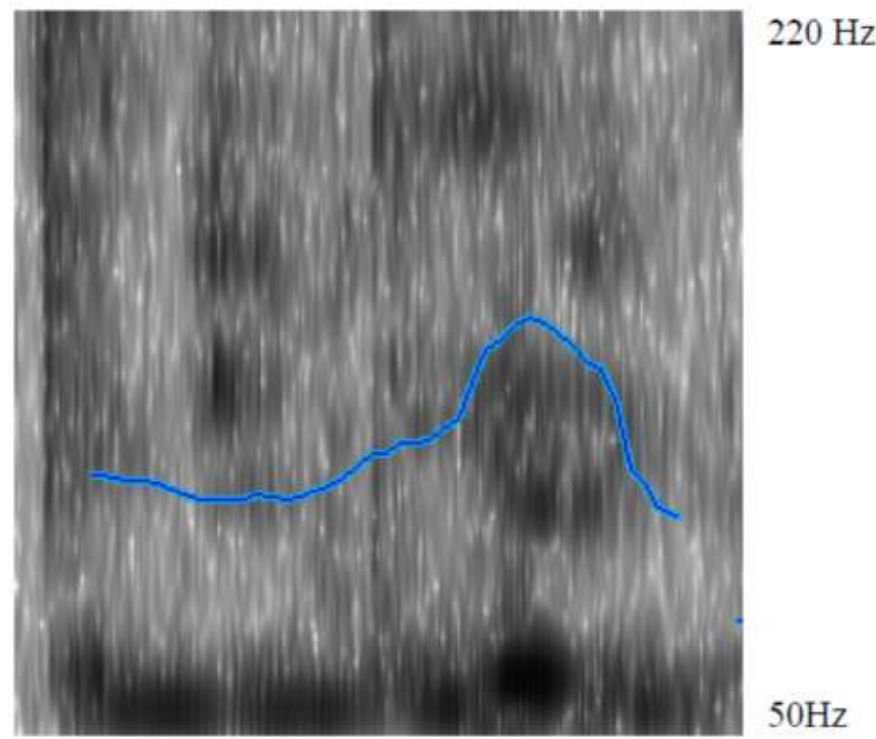

$$
\text { en } \quad \text { gi NEE ring }
$$

(Tonique de tête basse, rise-fall [107,8 Hz - 149,4 Hz - 104 Hz])

Fig. 2 : Ton nucléaire fréquent: rise-slump

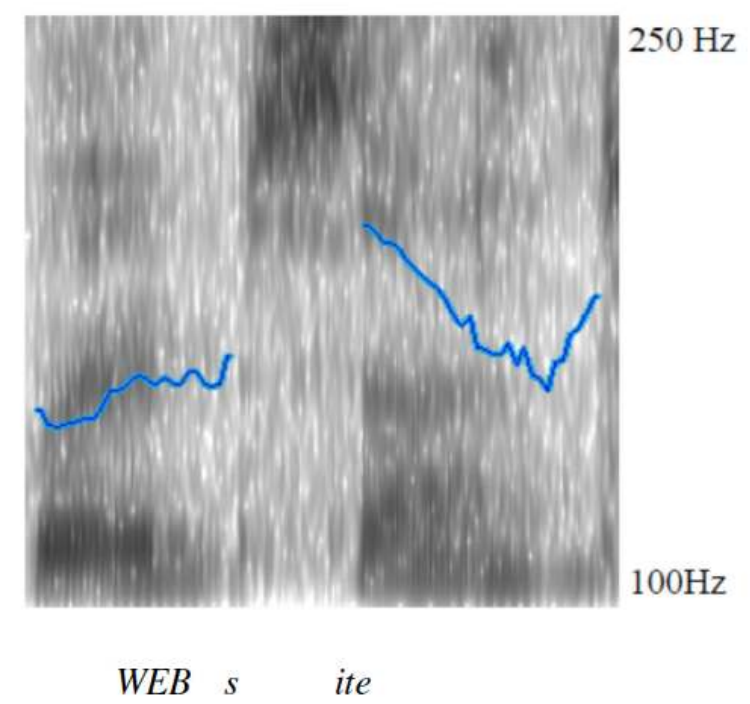

(Atones initiales basses, rise [“Jump up"]-slump [146,1 Hz-195,9 Hz-164,7 Hz])

Ces tons sont caractéristiques d'un système intonatif observé dans plusieurs centres urbains du nord de la Grande-Bretagne et nommé par Cruttenden $(1994 ; 2001$; 2007) UNBI (Urban North British Intonation) (Wilhelm, 2015). Cruttenden (2007:271) définit ce sytème de la façon suivante : « an intonational system that operates in a number of cities in Northern Britain [...] characterised by a default intonation involving rising or risingslumping nuclear pitch patterns ». Nous reviendrons plus tard sur les raisons de la 
présence d'un système associé aux conurbations urbaines à Lewis et Harris, majoritairement rurales.

\subsubsection{La qualité de voix} dans les tranches d'âge supérieures. Ceci est particulièrement frappant chez les adolescents, dont la production se caractérise par de nombreuses innovations phonétiques et par la régression de plusieurs traits saillants des accents traditionnels. Ces éléments semblent signaler un changement en cours.

Pour comprendre les raisons de cette évolution, il faut considérer au moins deux facteurs. Le premier est le déclin du gaélique et par conséquent de son influence sur l'anglais de Lewis et Harris. Le second est l'accroissement de la mobilité géographique dans le contexte hébridéen.

\subsection{Facteurs à l'origine de l'évolution des variétés d'anglais en usage à Lewis et Harris}

\subsubsection{Le déclin du gaélique}

Suite entre autres aux efforts déployés par les autorités éducatives pour réprimer l'usage du gaélique dans les Iles Hébrides (voir l'introduction), l'usage de celui-ci a considérablement régressé à l'école, dans les foyers et dans les situations courantes, comme en témoignent les données rassemblées dans le tableau 1.

Tableau 1 : Proportion de locuteurs hébridéens capables de s'exprimer en gaélique, de 1971 à 2001

\begin{tabular}{|l|l|}
\hline 1971 & $81,6 \%$ \\
\hline 1981 & $80,0 \%$ \\
\hline
\end{tabular}

Corela, 16-1 | 2018 


\begin{tabular}{|l|l|}
\hline 1991 & $68,9 \%$ \\
\hline 2001 & $59,6 \%$ \\
\hline 2011 & $40 \%(?)$ \\
\hline
\end{tabular}

Source : Recensements nationaux (Sites Scotland's Census et SCROL)

Ce phénomène est particulièrement sensible dans la zone urbaine de Stornoway (fig. 3). On observe un éphémère renversement de tendance dans les années 1980. Celui-ci coïncide avec une tentative de promouvoir à nouveau l'enseignement en gaélique dans les écoles hébridéennes. Cette inversion n'eut cependant rien de durable, vraisemblablement en raison de l'abandon du gaélique dans les foyers. Le déclin reprend et s'accentue à partir des années 1990.

Fig. 3 : Proportion de la population de Stornoway capable de comprendre ou de parler gaélique par année de naissance, de 1910 aux années 2000 (de droite à gauche), \%

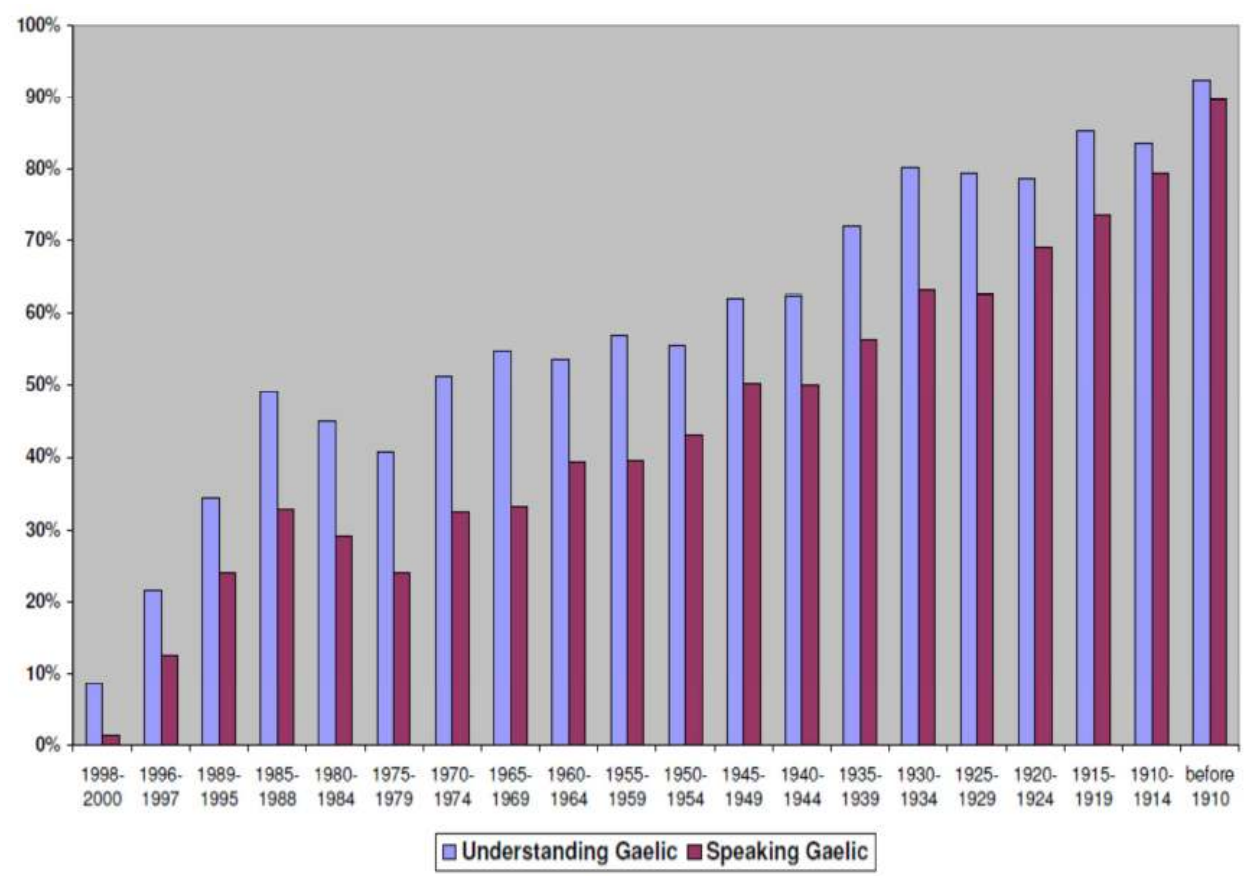

(Source: Gaidhlig Local Studies, Vol. 15 [élaboré à partir des données du recensement de 2001])

\subsubsection{Une mobilité géographique accrue}

Divers facteurs socioculturels ont conduit à une situation de mobilité géographique accrue dans les Iles Hébrides. De nombreux départs doivent être notamment attribués aux études suivies par les jeunes locuteurs en Écosse continentale. Ces dernières années, les mouvements de population vers ou depuis Lewis et Harris concernent essentiellement des individus âgés de 15 à 30 ans (fig. 4). Or, la partie inférieure de cette tranche d'âge est celle dans laquelle les modifications les plus importantes sont susceptibles d'intervenir dans l'idiolecte des locuteurs (Chambers and Trudgill, 1998 :77-79, 152-159). On conçoit aisément que le contact avec d'autres communautés linguistiques induit par ces 
déplacements conduise à des évolutions de l'anglais parlé par es insulaires eux-mêmes. Les éléments extérieurs transmettent en effet certains traits de leur variété d'origine, et les locuteurs autochtones, lorsqu'ils reviennent d'Écosse continentale, agissent comme des «missionnaires linguistiques» (Steinsholt , 1962, in Trudgill, 1986:56-57), introduisant dans les îles des innovations empruntées aux locuteurs des métropoles où ils résident désormais.

Fig. 4 : Flux migratoires vers et depuis les Hébrides extérieures par âge (en \% de la population) 2009-2011

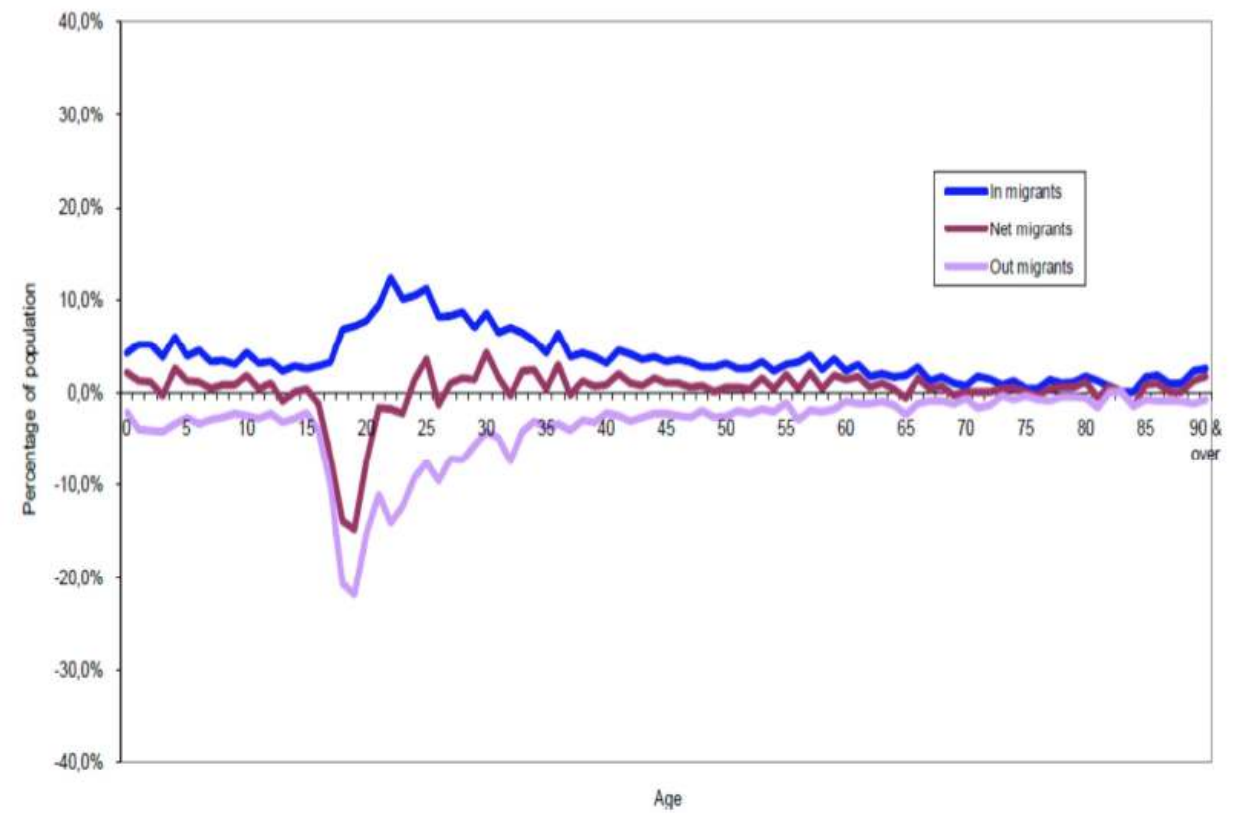

(Source : GRO Scotland)

\subsection{Innovations remarquables}

\subsubsection{Consonnes}

51 Comme dans la plupart des régions de Grande-Bretagne, les innovations les plus remarquables concernent la réalisation phonétique des phonèmes consonantiques. Kerswill (2003:231) définit d'ailleurs les consonnes comme les fers de lance de la diffusion phonétique (« the torchbearers of geographical diffusion »).

\subsubsection{Le « remplacement glottal » de /t/}

Il s'agit de la réalisation de /t/ par un coup de glotte: [?]. Ce type de variantes est aujourd'hui observable dans la quasi-totalité des accents de l'anglais britannique, et ce dans tous les environnements phonétiques excepté - en principe - à l'initiale ${ }^{13}$. A Lewis et Harris, le remplacement glottal de / $t /$ est abondamment observé dans la production des jeunes locuteurs, particulièrement celle des adolescents (right off $=>$ [uaI̦?'ơf] ; I'm waiting $\Rightarrow$ [am'we $\left.{ }^{\mathrm{I}} \mathrm{I} \mathrm{I}\right]$ ]; Saturday $\Rightarrow>$ ['sa?əde]), même lors de la lecture de certains segments inclus dans la liste de mots (Finlay's go [?] a brother) alors qu'il est notablement absent de 
celle des locuteurs les plus âgés. Dans les années 1980, Shuken (1984:158) observait des phénomènes similaires à Skye, mais non dans les Hébrides extérieures.

Une autre variante innovante de / $\mathrm{t} /$ en position intervocalique est la battue alvéolaire

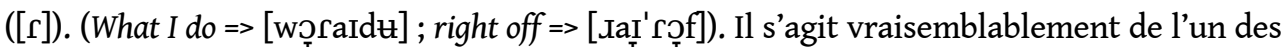
traits qui amènent certains locuteurs âgés à déplorer que les jeunes Hébridéens s'expriment aujourd'hui comme des Américains. À la vérité, la piste d'une influence américaine semble hautement improbable. En l'absence de données précises sur la diffusion de la réalisation de / $\mathrm{t} /$ comme une battue alvéolaire en Écosse continentale, nous nous bornerons à souligner que cette variante permet une indéniable économie articulatoire (Harris \& Kaye, 1990), ce qui explique entre autres son emploi en anglais américain (Wells, 1982: 248-251), mais aussi traditionnellement dans le nord de l'Angleterre (Wells, 1982 : 370) et, aujourd'hui, en divers endroits du sud de la GrandeBretagne (Wilhelm, 2005 : 159).

\subsubsection{Variantes innovantes $\mathrm{de} / \theta /$ et $/ \delta /$ en position intervocalique}

$\mathrm{Si}$, dans la plupart des cas, les locuteurs les plus âgés et les plus conservateurs utilisent des fricatives dentales pour réaliser $/ \theta /$ et /ð/, c'est-à-dire les variantes «standard » de ces phonèmes ${ }^{14}$, en position intervocalique, il est fréquent d'observer chez les adolescents deux types de réalisations innovantes de /ð/: la battue alvéolaire [ $[$ ] et la suppression

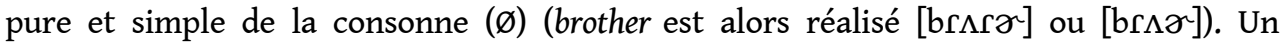
phénomène similaire était observé à Glasgow dans les années 1980 (McAfee, 1983 :33, in Stuart-Smith, 1999: 209), et il est vraisemblable que ces variantes se soient diffusées plus ou moins indirectement depuis cette métropole.

55 Aucune trace d'une antériorisation de $/ \theta /$ et /ð/ semblable à celle que Timmins, Tweedie \& Stuart-Smith (2004) observaient à Glasgow dans les années 2000 n'apparaît dans les données collectées en 2005, et nous n'avons pas noté d'occurrence de ce phénomène au cours de nos observations informelles. Des remarques émises par au moins un locuteur hébridéen (non linguiste) connu de l'auteur depuis de nombreuses années suggère cependant que des variantes de ce type apparaissent dans la production de certains adolescents. Une fois encore, il est tentant d'envisager que des processus de diffusion géographique " hiérarchique » (Britain, 2005) aient pu opérer, Glasgow jouant un rôle clef dans ce phénomène en raison de son importance démographique.

\subsubsection{Variantes vélaires et vocaliques de /l/}

On l'a vu plus haut (2.2.8.), les accents traditionnels de Lewis et Harris se caractérisent par des variantes claires de / $/$ dans tous les environnements phonétiques. On assiste pourtant à la diffusion progressive de variantes vélaires ou vocalisées en position

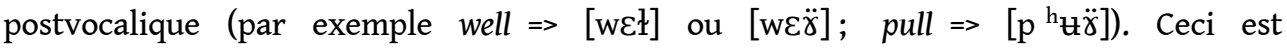
essentiellement vrai dans la production des locuteurs les plus jeunes, mais ce phénomène est également observé chez certains locuteurs plus âgés.

Certaines variantes observées dans la production des adolescents hébridéens pourraient être apparentées à ce que Stuart-Smith et al. (2004: 5-7) appellent le 'Scots Lvocalisation', phénomène dont la manifestation contemporaine constitue le prolongement de processus à l'œuvre depuis le quinzième siècle (Stuart-Smith et al. $2004: 7$ ). Ce type de vocalisation n'est observée qu'après les voyelles /a/, /o/, /u/ (Stuart- 
Smith et al. $2004: 15)$. /1/ est alors réalisé avec des traits phonétiques en tout point similaires à ceux de la voyelle qui le précède (Stuart-Smith, communication personnelle). Les enregistrements réalisés en 2005 comportent ainsi une occurrence de football => ['fł?bo:] et des occurrences de Paul => [po:]. Les variantes vélaires ou les variantes vocalisées qui impliquent la réalisation d'une voyelle d'arrière ou une voyelle centrale distincte du son qui la précède (voir paragraphe ci-dessus) ont vraisemblablement une autre origine.

Certains considèrent que les variantes vocalisées de / $/$ récemment observées dans divers accents de l'anglais britannique ont pour origine l'accent Cockney ou du moins la ville de Londres. Une hypothèse similaire est parfois émise pour rendre compte de la diffusion des réalisations glottales de $/ \mathrm{t} /$ ou de l'antériorisation de $/ \theta /$ et $/ \delta /$. Bien qu'aucune donnée empirique ne permette d'infirmer totalement cette proposition, de nombreux linguistes se montrent toutefois sceptiques quant à sa validité (Beal, Britain, Daniels, communications personnelles ; voir également Wilhelm 2012 : 9-10).

\subsubsection{Voyelles : l'antériorisation de FOOT et de GOOSE}

On note chez les jeunes locuteurs une nette tendance à l'antériorisation de FOOT et de GOOSE, moins présente dans la production des locuteurs les plus âgés (fig. 5 et 6).

Fig. 5 Spectrogramme des mots books, boot, goose et good prononcés par Donald, 73 ans

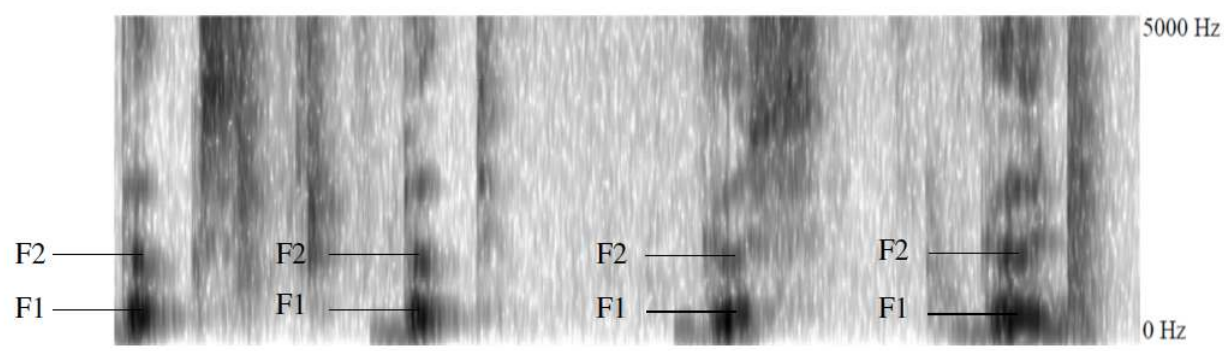

Fig. 6 Spectrogramme des mots books, boot, goose et good prononcés par Sean, 16 ans

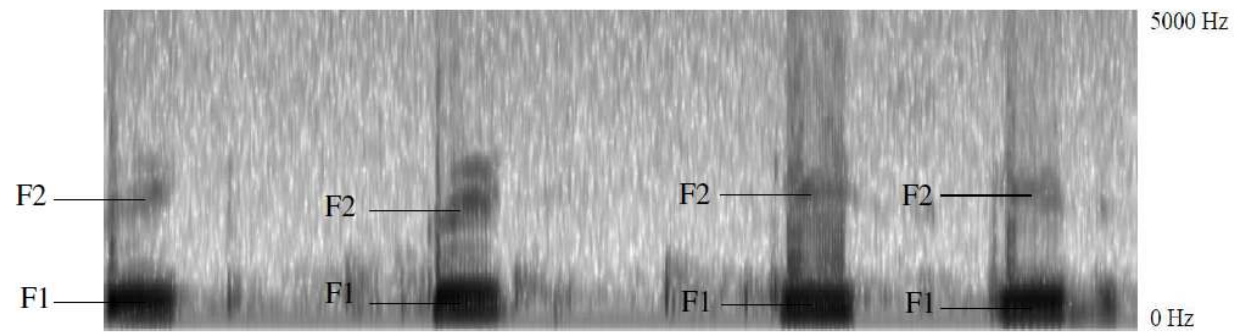

La fig. 5 laisse apparaitre le fait que le second formant (F2) ${ }^{15}$ des voyelles de FOOT et de GOOSE est bas et assez proche de F1 dans la production de Donald. Ceci témoigne d'une relative rétraction de ces voyelles (en anglais écossais, GOOSE est ordinairement centrale ([u]), même chez les locuteurs les plus conservateurs). Dans la production de Sean (fig. 6), l'élévation bien plus marquée de F2 et son éloignement de F1 signale leur antériorisation. 


\subsection{Le nivellement dialectal}

61 Le nivellement dialectal (dialect levelling) consiste en la disparition plus ou moins progressive des variantes les plus marquées dans une zone géographique donnée (Trudgill, 1986 : 98).

Le nombre restreint d'occurrences des variables présentes dans les données recueillies auprès des adolescents (voir section 1.2.) n'étant pas statistiquement représentatif, le tableau 2 n'a pas été élaboré à partir de données quantifiées. Il rassemble les phénomènes répertoriés au cours de seize années d'observations informelles et non à partir de l'étude exclusive des statistiques élaborées à partir des corpus dont nous disposons.

Tableau 2 : Régression de certaines variantes à Lewis et Harris

\begin{tabular}{|c|c|}
\hline Variantes résistantes & Variantes en régression \\
\hline$/ \mathrm{x} /$ & $\begin{array}{l}\text { Préaspiration des plosives } \\
\text { sourdes }\end{array}$ \\
\hline $\begin{array}{l}\text { Palatalisation de /s/ dans la suite /st/ avant les voyelles } \\
\text { fermées d'avant }\end{array}$ & $\begin{array}{l}\text { Dévoisement des consonnes } \\
\text { voisées }\end{array}$ \\
\hline & Opposition /w/ - /M/ \\
\hline & $\begin{array}{l}\text { Insertion de schwas } \\
\text { épenthétiques }\end{array}$ \\
\hline & $\begin{array}{l}\text { Rétroflexion de /s/ et /z/ après } \\
/ \mathrm{r} /\end{array}$ \\
\hline & Présence de /r/ postvocalique \\
\hline
\end{tabular}

Le nivellement dialectal s'explique par le contact entre diverses variétés géographiques. Il pourrait paraître peu étonnant que le phonème $/ \mathrm{x} /$ résiste au nivellement dans la mesure où il existe dans la plupart des accents écossais. L'attrition progressive de cette variante chez les jeunes locuteurs de Glasgow (Stuart-Smith \& Lawson, 1999) pourrait cependant constituer le signe précurseur d'un changement plus généralisé. Quoi qu'il en soit, aucun changement de cette nature n'est perceptible à ce jour en anglais hébridéen.

La palatalisation de /s/ dans l'agrégat /st/ est aujourd'hui observée avant les voyelles fermées d'avant dans de nombreux accents de l'anglais (Glain, 2012), et son usage de plus en plus répandu pourrait contribuer à expliquer que l'accroissement de la mobilité géographique n'affecte pas l'emploi de cette variante à Lewis et Harris.

Aux évolutions les plus marquantes observées dans la production des adolescents s'ajoute la régression des occurrences de $/ \mathrm{r} /$ en position finale et/ou préconsonantique, qui parait signaler un déclin naissant de la rhoticité (chez plusieurs adolescents, on entend des

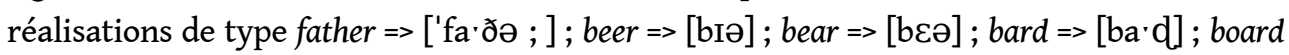
$\Rightarrow$ [bo'd]; farmers ['fa'məz]). Here est systématiquement réalisé [(h)Iə]/[(h)re] par les adolescents rencontrés dans la rue. Cette prononciation se caractérise par une absence 
totale de $/ \mathrm{r}$ / et une diphtongue au second élément extrêmement ouvert. On notera toutefois que ces mots et d'autres unités lexicales comportant un $/ r /$ final ou préconsonantique sont cependant parfois toujours réalisés par les jeunes locuteurs

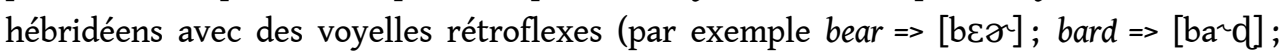
board $=>$ [bフ^d), reliques de la présence de $/ \mathrm{r} /$ (voir section 2.2.6.).

Le déclin de la rhoticité de l'anglais écossais est observé depuis longtemps à Glasgow, particulièrement dans la production de locuteurs de milieu ouvrier (Trotter 1901, in Stuart-Smith et al. 2014). Romaine (1978) et Johnston (1985) recensèrent le phénomène à Édimbourg dans les années 1970 et 1980. À Lewis et Harris, ce déclin est essentiellement perceptible à Stornoway, où certains adolescents semblent aussi plus enclins à utiliser des approximantes post-alvéolaires/rétroflexes de $/ \mathrm{r} /$ là où les locuteurs plus traditionnels préfèrent la battue alvéolaire ou la trille (ceci rejoint les observations réalisées par Romaine [1978] à Édimbourg.)

Un autre signe du déclin de la rhoticité consiste en l'accentuation, chez certains jeunes locuteurs, du phénomène connu sous le nom de pre-/r/ breaking (Wells 1982: 213-218), c'est-à-dire la diphtongaison centralisante des voyelles qui précèdent $/ \mathrm{r} /$.

Les fig. 7 et 8 montrent des spectrogrammes des mots beer et bear, respectivement réalisés

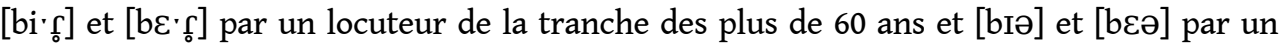
adolescent. Diachroniquement, c'est l'occurrence de ce phénomène qui explique la présence de diphtongues à la fin de mots semblables dans la $R P$, non rhotique.

Fig. 7 : Spectrogramme des mots beer et bear prononcés par Marietta, 72 ans

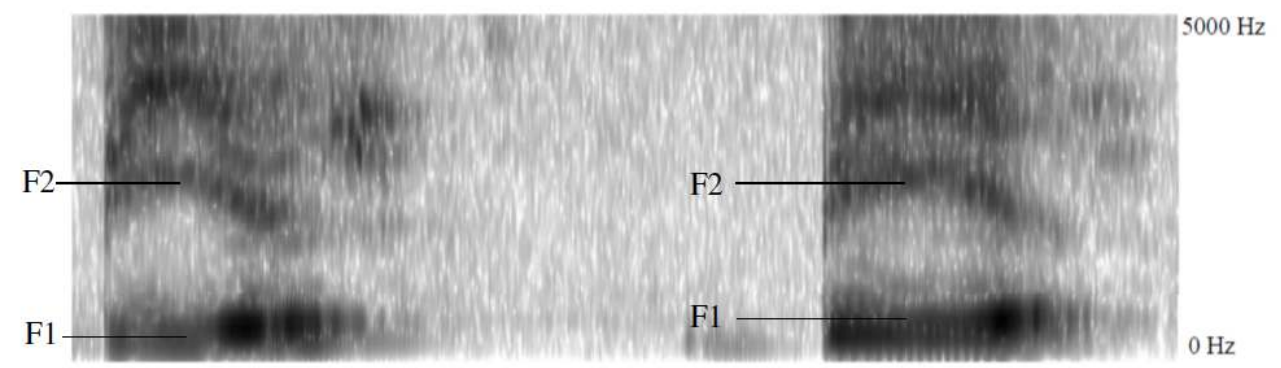

Fig. 8 : Spectrogramme des mots beer et bear prononcés par Sean, 16 ans

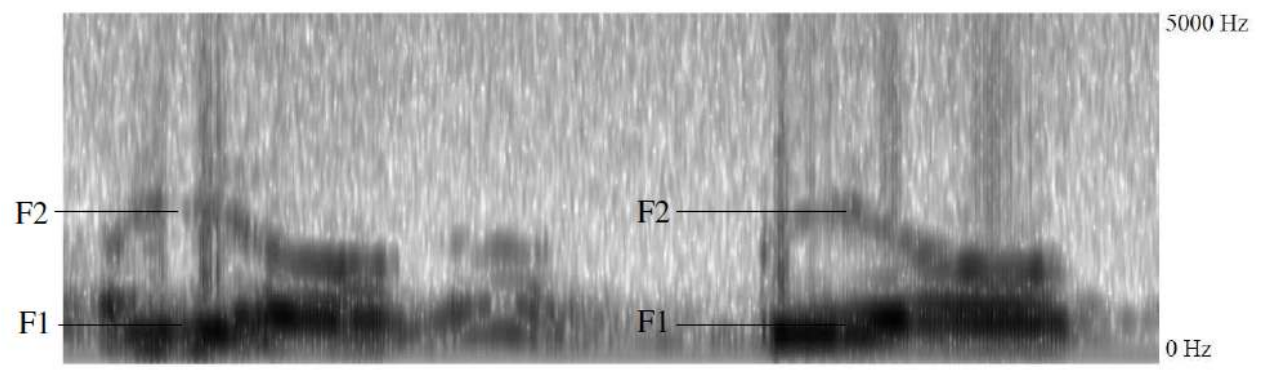

Un examen de la fig. 7 laisse apparaître un faible rapprochement des premiers formants des voyelles de beer et bear (F1 et F2) dans la production de Marietta au fur et à mesure que le phonème est réalisé, ce qui témoigne d'un faible degré de diphtongaison. Dans la production de Sean, en revanche (fig. 8), F1 et F2 se rapprochent très nettement, ce qui témoigne d'une diphtongaison marquée. 
70 Bien que la quantité de données présentes dans les enregistrements réalisés dans la rue (voir section 1.2.) soit trop faible pour en tirer des généralisations, notre impression est que les signes de perte de la rhoticité observée à Lewis et Harris sont moins marqués que ceux qui apparaissent aujourd'hui à Glasgow (cf. Stuart-Smith et al. 2014). La situation évoque cependant à plusieurs égards celle qui prévaut dans cette grande agglomération et ses environs.

71 En premier lieu, il existe en effet dans la production des adolescents à Stornoway un continuum phonétique dans la réalisation $\mathrm{du} / \mathrm{r} /$ postvocalique. À une extrémité de ce gradient, on trouve des consonnes variablement dévoisées semblables à celles que l'on observe chez les locuteurs des tranches d'âges supérieures (voir section 2.2.6.). À l'autre, on observe une absence totale de phone consonantique. Entre ces deux pôles apparaissent des voyelles variablement rétroflexes et des approximantes inégalement perceptibles. La présence de consonnes rétroflexes à la fin de syllabes comportant en coda des suites graphiques de type $<\mathrm{r}+$ Consonne alvéolaire $>$ constitue également un signe de la présence sous-jacente de /r/ lorsque ce phonème n'est pas réalisé.

72 Enfin, à l'instar de ce qui se produit à Glasgow, le continuum observé dans les Iles Hébrides dans la réalisation $\mathrm{du} / \mathrm{r} /$ postvocalique intègre une composante sociologique déterminante. Alors que les jeunes gens qui adoptent une attitude favorable envers la communauté hébridéenne semblent favoriser les variantes rhotiques, les variantes nonrhotiques paraissent majoritairement être employées par des adolescents qui en rejettent les valeurs traditionnelles ou souhaitent s'en démarquer (Wilhelm, 2005 : 85-86). Ceci suggère que la perte de la rhoticité jouit avant tout d'un prestige " couvert " (Labov [1966] 2006 : 402). Quoi qu'il en soit, les éléments recensés ici peuvent légitimement conduire à s'interroger sur l'avenir de la rhoticité des accents écossais.

\subsection{Quelques pistes de réflexion}

73 Dans un contexte de déclin du gaélique et de mobilité géographique accrue, nous observons une évolution marquée des accents de l'anglais de Lewis et Harris. Pour le sociophonéticien, cette évolution n'est pas sans soulever des questions plus générales, et c'est sur ce type de considérations que nous nous concentrerons pour finir.

\subsubsection{Facteurs favorisant la diffusion étendue de certaines variantes phonétiques}

74 L'évolution des accents de Lewis et Harris témoigne dans une zone géographique relativement isolée de la présence récente d'innovations phonétiques observées à grande échelle dans le contexte britannique (Wilhelm, 2011). Il s'agit notamment de la glottalisation de / $t$ / et de la vocalisation de /l/. Comment expliquer l'étendue de la diffusion de ces variantes? Nous proposons ici quelques hypothèses :

La diffusion de réalisation glottale de /t/ s'explique entre autres par sa saillance marquée. Selon Trudgill (1986: 12-14), une innovation linguistique doit en effet être saillante pour se diffuser largement. Les réalisations glottales de / $t /$ semblent appréciées des adolescents car elles leur permettent de se démarquer des locuteurs plus âgés et peutêtre de s'identifier à la jeunesse des grandes agglomérations.

76 La présence des variantes «non-standard» de / $\theta /$ et /ð/ (battue alvéolaire ou élision en position intervocalique - voir section 3.2.1.2.) pourrait être favorisée par les mêmes facteurs, mais aussi par le fait que la réalisation canonique de ces phonèmes requiert un 
effort important de précision articulatoire. C'est pourquoi les enfants éprouvent fréquemment des difficultés à acquérir ces consonnes, recensées dans peu de langues du monde. Au cas où la diffusion de variantes antériorisées de de $/ \theta /$ et $/ ð /$ se confirmerait en anglais hébridéen (voir section 3.2.1.2.), un scénario de diffusion directe ou indirecte depuis Glasgow constituerait une piste privilégiée (voir section 3.2.1.3.). L'hypothèse londonienne paraît davantage sujette à caution (voir section 3.2.1.).

La diffusion de la vocalisation de $/ 1 /$, phénomène récurrent dans l'histoire de l'anglais et d'autres langues du monde, s'explique vraisemblablement par l'économie articulatoire que cette innovation permet de réaliser ainsi que par son caractère non-marqué (Johnson \& Britain 2002: 302-304). L'approximante latérale possède en effet bien des points communs avec une voyelle et sa vocalisation se produit fréquemment lors de l'acquisition de la langue maternelle (Johnson \& Britain 2002: 303-304). La diffusion de ce type de variantes constitue une exception à la règle selon laquelle le succès d'une innovation phonétique dépend entre autre de sa saillance (cf. Trudgill, 1986 : 12-14).

\subsubsection{Les dimensions du changement linguistique} l'égard de la vie insulaire (Wilhelm, 2005 : 85-86; voir également la section 3.3.). Ils n'en sont que plus prompts à adopter les innovations linguistiques qu'ils associent plus ou moins consciemment aux valeurs traditionnelles de leur communauté et à se départir des spécificités qui les désignent comme Hébridéens. C'est la dimension socio-psychologique du changement linguistique.

81 Enfin, l'amélioration du réseau routier et le développement des moyens de transport favorisent les échanges entre locuteurs et précipitent l'évolution des accents. De tels facteurs relèvent de la dimension spatiale du changement linguistique.

\subsubsection{La qualité de voix en tant que marqueur de spécificité dialectale} chaque langue du monde (Laver, 1994; Coadou, 2007). Ceci s'applique également aux divers dialectes d'une langue donnée. Or, ce domaine de recherche demeure largement sous-exploré. Le cas de l'anglais de Lewis et Harris, variété géographique fréquemment caractérisée entre autres par une qualité de voix résultant d'un positionnement rétracté de la pointe de la langue (voir section 2.3.2), encourage pourtant à ne pas négliger l'étude de ce trait prosodique en tant que marqueur de spécificité dialectale.

\subsubsection{Un nouvel éclairage sur les origines d'UNBI ?}

83 La présence du système intonatif UNBI dans plusieurs villes du nord du Royaume-Uni a suscité bien des spéculations sur ses origines. L'hypothèse d'une origine celtique a été émise dès 1975 par Knowles (1975), puis reprise par Cruttenden, avec des réserves 
(Cruttenden, 1994 : 160). Alors que les travaux de Dalton \& Ní Chasaide (2003) sur certains dialectes de l'anglais d'Irlande et de l'irlandais semblent corroborer cette proposition, Hirst (2008) pense pour sa part qu'UNBI pourrait trouver ses racines dans la langue des Vikings.

Le cas de l'anglais de Lewis pourrait tout d'abord sembler militer en faveur d'une origine celtique. En effet, le système intonatif des variétés de gaélique locales est remarquablement similaire à celui de l'anglais parlé dans l'île, et il y a tout lieu de penser que l'intonation de l'anglais de Lewis et Harris est calquée sur celle du gaélique de ces localités. Il est notamment frappant de noter que le gaélique de Lewis fait appel à un ton de base de type rise-fall semblable à celui qui caractérise l'anglais de Lewis et Harris (fig. 9, voir section 2.3.1.), ce qui renforce l'hypothèse celte.

Fig. 9 : Gaélique de Lewis - tons nucléaires de base de type rise-fall (Donald, 73 ans)

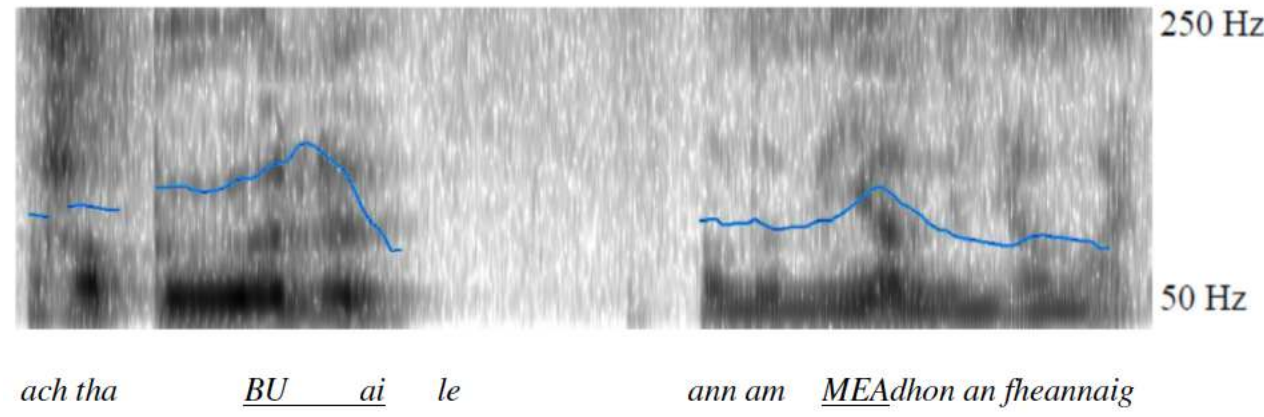

(but it had an impact on the middle of the lazybed)

Il ne faut cependant pas oublier que Lewis et Harris, les îles les plus septentrionales des Hébrides extérieures, ont subi par le passé l'influence des peuplades nordiques suite aux invasions vikings. Cette influence est encore aujourd'hui très perceptible, notamment sur les plans culturel et toponymique (voir par exemple Shorn \& Quinn, 2014). Du reste, une comparaison attentive des zones des îles Britanniques où UNBI est attesté et des localités où les Vikings se sont établis entre le VIII ${ }^{\mathrm{e}}$ et le $\mathrm{X}^{\mathrm{e}}$ siècle fait apparaitre de surprenantes correspondances (Hirst, 2009; Wilhelm, 2015). Les hypothèses celtique et scandinave pourraient ne pas s'avérer contradictoires, mais complémentaires. On peut en effet imaginer que certains traits intonatifs se soient diffusés directement dans l'anglais des zones anglophones et indirectement par le biais du gaélique, dans celui des zones de Gaélie exposées aux invasions vikings ${ }^{16}$.

\subsection{Conclusion sous forme de synthèse}

Un examen des spécificités phonétiques et phonologiques de l'anglais de Lewis et Harris met en évidence les traits celtiques de cette variété de contact façonnée par le gaélique hébridéen. Ces spécificités sont observées tant au niveau segmental que suprasegmental, ce qui souligne le statut essentiel des éléments prosodiques dans l'étude de la variation dialectale.

Le déclin du gaélique favorise une transformation rapide des accents de l'anglais hébridéen, phénomène renforcé par divers facteurs géographiques et sociopsychologiques. La diffusion de diverses innovations phonétiques à grande échelle et la 
régression de plusieurs spécificités locales sont particulièrement remarquables dans la production des jeunes locuteurs, notablement celle des adolescents.

Le cas de l'anglais de Lewis et Harris présente en soi un intérêt certain. Il fournit en outre un éclairage opportun sur des questions linguistiques plus larges, permettant par exemple de mieux appréhender certains des facteurs qui favorisent la diffusion d'innovations phonétiques. Il met également en lumière l'importance de la prise en compte des éléments prosodiques dans l'étude de la variation dialectale. Sur un plan diachronique, il apporte enfin des informations pertinentes dans le cadre du débat sur l'origine d'UNBI, le système intonatif observé dans de nombreuses localités du nord du Royaume-Uni. Loin d'être contradictoires, les hypothèses de ses origines scandinave ou celtique pourraient mettre en lumière un double phénomène : la diffusion directe d'UNBI dans les dialectes de l'anglais des zones anglophones exposées aux invasions vikings et celle, indirecte, par le biais des langues celtes, du même système intonatif dans ceux des zones celtophones soumises à l'influence nordique.

\section{Remerciements}

L'auteur tient à exprimer ses remerciements les plus chaleureux à Cairistiona NicCoinnich (University of the Highlands and Islands) et Sandra Corbett (University of Edinburgh) pour leur aide lors de la transcription des enregistrements des dialogues en gaélique.

\section{BIBLIOGRAPHIE}

BIRD Barbara, 1997, 'Past and present studies of Hebridean English phonology', in H. TRISTRAM (ed.), The Celtic Englishes, Heidelberg, Winter, pp. 287-300.

BLACK Ronald, 1992, Cothrom Ionnsachaidh, Édimbourg, University of Edinburgh, Department of Celtic Edition.

BRITAIN David, 2005, 'Geolinguistics and Linguistic Diffusion', in U. AMMON and al. (eds) Sociolinguistics: International Handbook of the science of Language and Society. Berlin: Mouton de Gruyter, pp. 34-48.

CLEMENT R. D, 1984, 'Gaelic', in P. TRUDGILL (ed.), Language in the British Isles, Cambridge, Cambridge University Press, pp. 318-342.

CHAMBERS Jack \& TRUDGILL Peter, 1998 (1980), Dialectology (2 ${ }^{\text {nd }}$ ed.), Cambridge, Cambridge University Press.

COADOU Marion, 2007, Qualité de voix et accents régionaux en anglais britannique, thèse de doctorat non publiée, Laboratoire Parole et Langage (LPL), Aix-en-Provence, Université de Provence.

CRUTTENDEN Alan, 1994, 'Rises in English', in W. J. LEWIS (ed.), Studies in General and English Phonetics: Essays in Honour of Professor J. D. O'Connor, Londres, Routledge, pp. 155-173. 
CRUTTENDEN Alan, 1997 (1986), Intonation (2 $2^{\text {nd }}$ ed.), Cambridge, Cambridge University Press.

CRUTTENDEN Alan, 2001, 'Mancunian intonation and intonational representation', Phonetica 58, pp. 53-80.

CRUTTENDEN Alan, 2007, 'Intonational diglossia: a case study of Glasgow', Journal of the International Phonetic Association, vol. 37, n³, pp. 257-274.

DALTON Martha \& NÍ CHASAIDE Ailbhe, 2003, 'Modelling intonation in three Irish dialects', Proceedings of the $15^{\text {th }}$ International Congress of Phonetic Sciences, Barcelona, pp. 1073-1076.

GLAIN Olivier, 2012, « Les Cas de Palatalisation Contemporaine (CPC) en anglais », La Clé des langues (Lyon: ENS LYON/DGESCO), <http://cle.ens-lyon.fr/anglais/les-cas-de-palatalisationcontemporaine-cpc-en-anglais--145650.kjsp?RH=CDL_ANG120300>, consulté le 5 août 2013.

HARRIS J. and KAYE J., 1990, ‘A tale of two cities: London glottalling and New York City tapping', The Linguistic Review, 7, pp. 251-74.

HICKEY Raymond, 1999, 'Dublin English: current changes and their motivation', in P. FOULKES \& G. DOCHERTY (eds.), Urban Voices, Londres, Arnold, pp. 265-281.

HIRST Daniel, 2008, 'Could the rising intonation patterns of Urban Northern Britain be Nordic Prosody?', Nordic Prosody, University of Helsinki.

KERSWILL Paul, 2003, 'Dialect levelling and geographical diffusion in British English', in D. B RITAIN and J. CHESHIRE (eds.), Social Dialectology, Amsterdam, John Benjamins, pp. 223-243.

KNOWLES Gerald, 1975, Scouse: the Spoken Dialect of Liverpool, thèse de doctorat non publiée, University of Leeds.

LABOV William, (1966) 2006, The Social Stratification of English in New York, Cambridge, University of Cambridge Press.

LAVER John, 1994, Principles of Phonetics, Cambridge, Cambridge University Press.

MACDONALD Donald, 1978, Lewis: a History of the Island, Édimbourg, Gordon Wright.

ROBERTSON Boyd, 2011, Language, Culture and Community, transcription d'une allocution délivrée le 17 octobre 2011 à Stornoway, Kershader, The Islands Books Trust.

ROMAINE, Suzanne, 1978, 'Postvocalic /r/ in Scottish English: sound change in progress?', in P. T RUDGILL (ed.), Sociolinguistic patterns in British English, London, Edward Arnold, pp 144-57.

SHORN Britanny \& QUINN Judy (éds.), 2014, The Vikings in Lewis, Centre for the Study of the Viking Age, University of Nottingham, < http://www.nottingham.ac.uk/re-search/groups/csva/ documents/lmfpublications/lmf2-isleoflewis, thevikingsinlewis.pdf>

SHUKEN Cynthia, 1984, 'Hebridean English', in P. TRUDGILL (ed.), Language in the British Isles, Cambridge, Cambridge University Press, pp. 152-166.

STUART-SMITH Jane, 1999, 'Glasgow: accent and voice quality', in P. FOULKES \& G. DOCHERTY (eds.), Urban Voices, Londres, Arnold, pp. 203-222.

STUART-SMITH Jane \& LAWSON Eleanor, 1999, 'A sociophonetic investigation of the "Scottish" consonants $(/ \mathrm{x} /$ and $/ \mathrm{hw} /)$ in the speech of Glaswegian children, Proceedings of the XIVth International Congress of Phonetic Sciences, 1999, pp. 2541-2544.

STUART-SMITH Jane, LAWSON Eleanor \& SCOBBIE James .M. 2014, 'Derhoticisation in Scottish English: a sociophonetic journey, in CELATA, C. and CALAMAI, S. (eds.) Advances in Sociophonetics.

Amsterdam, John Benjamins, pp. 57-94. 
TIMMINS Claire, TWEEDIE Fiona \& STUART-SMITH Jane, 2004, The Glasgow Speech Project. Accent change in Glaswegian: A sociophonetic investigation (based on the 1997 corpus). University of Glasgow.

TRUDGILL Peter, 1986, Dialects in Contact, Oxford, Blackwell.

WELLS John, 1982, Accents of English, Cambridge, Cambridge University Press.

WILHELM Stephan, 2005, Accent Change in the British Isles: the Four Dimensions of an Invisible Hand, $\mathrm{m}$ émoire de maîtrise non publié, Université de Nancy 2.

WILHELM Stephan, 2011, « Réflexions sur quelques innovations phonétiques observées en anglais britannique », La Clé des Langues (Lyon : ENS LYON/DGESCO), <http://cle.ens-lyon.fr/anglais/ reflexions-sur-quelques-innovations-phonetiques-observees-en-anglais-britannique-134503.kjsp>, consulté le 23 mai 2018.

WILHELM Stephan, 2015, « Urban North British Intonation : Le système intonatif des accents de l'anglais du nord du Royaume-Uni », Actes du Colloque du 17 mai 2014, Les Amis du Crelingua. Paris : Les Amis du Crelingua, pp. 72-88.

WILLIAMS Ann \& KERSWILL Paul, 1999, 'Dialect levelling: change and continuity in Milton Keynes, Reading and Hull', in P. FOULKES \& G. DOCHERTY (ed.), Urban Voices, Londres, Arnold, pp. 141-162.

Pages web / sources en ligne :

Gaidhlig Local Studies <http://www.linguae-celticae.org>.

Scotland's Census <http://www.scotlandscensus.gov.uk/>

SCROL (Scotland's Census Results Online) http://www.scrol.gov.uk

\section{ANNEXES}

APPENDICE

Liste de mots proposée aux informateurs lors des enregistrements réalisés en 2005

\begin{tabular}{|l|l|l|}
\hline 1) PIT & 41) WEIGHT & 81) FORCE \\
\hline 2) PET & 42) POOR & 82) LOCH \\
\hline 3) PAT & 43) POUR & 83) BELLS \\
\hline 4) PUT & 44) PORE & 84) PILOT \\
\hline 5) PUTT & 45) PAW & 85) COMMA \\
\hline 6) POT & 46) TIDE & 86) YEARS \\
\hline 7) BEE & 47) TIED & 87) SMOOTH \\
\hline 8) BAY & 48) PAUSE & 88) BOOTH \\
\hline 9) BUY & 49) PAWS & 89) DRIVERS \\
\hline
\end{tabular}




\begin{tabular}{|c|c|c|}
\hline 10) BOY & 50) MEET & 90) FARMERS \\
\hline 11) ВоОТ & 51) MEAT & 91) SOMETHING \\
\hline 12) BOAT & 52) MATE & 92) MOUTH \\
\hline 13) BOUT & 53) THIN & 93) PETROL \\
\hline 14) BEER & 54) THICK & 94) FILM \\
\hline 15) BEAR & 55) PALM & 95) CHARLES \\
\hline 16) BIRD & 56) BALM & 96) cows \\
\hline 17) BARD & 57) BETTER & 97) PLEASURE \\
\hline 18) BOARD & 58) BITTER & 98) ROUGE \\
\hline 19) CITY & 59) BUTTER & 99) ENJOYING \\
\hline 20) SEEDY & 60) BATTER & QUITE GOOD \\
\hline 21) HAT & 61) BROTHER & QUITE NICE \\
\hline 22) DANCE & 62) FATHER & QUITE DIFFICULT \\
\hline 23) DAFT & 63) FOOTBALL & QUITE EASY \\
\hline 24) HALF & 64) MILK & I'VE GOT IT \\
\hline 25) FATHER & 65) BULK & FINLAY`S GOT A BROTHER \\
\hline 26) FARTHER & 66) SAM & CALUM`S GOT A COLD \\
\hline 27) PULL & 67) PSALM & \\
\hline 28) POOL & 68) WITCH & \\
\hline 29) POLE & 69) WHICH & \\
\hline 30) PAUL & 70) $\mathrm{BOOK}$ & \\
\hline 31) DOLL & 71) Воот & \\
\hline 32) $\mathrm{COT}$ & 72) GOOSE & \\
\hline 33) CAUGHT & 73) GOOD & \\
\hline 34) FIR & 74) НАРPY & \\
\hline 35) FERN & 75) GREEDY & \\
\hline 36) FUR & 76) MERCY & \\
\hline
\end{tabular}




\begin{tabular}{|l|l|l|}
\hline 37) FAIR & 77) BABY & \\
\hline 38) NOSE & 78) STUDENT & \\
\hline 39) KNOWS & 79) SPORT & \\
\hline 40) PLATE & 80) FURZE & \\
\hline
\end{tabular}

\section{NOTES}

1. $\mathrm{Au} \mathrm{XIX}^{\mathrm{e}}$ siècle, la plupart des associations chrétiennes et les écoles dirigées par des organisations tentèrent au contraire de promouvoir l'usage du gaélique (Robertson, 2011 : 27).

2. Voir par exemple Chambers \&Trudgill $1996: 76$.

3. L'inconvénient de l'adoption d'un protocole différent pour enregistrer les adolescents résidait en le fait que celle-ci accroissait la difficulté à évaluer le rôle du registre linguistique dans les contrastes observés avec les informateurs des tranches d'âge supérieures. Une tendance à la stratification des données émergeait toutefois nettement.

4. «[These] keywords [are] intended to be unmistakable no matter what accent one says them in. » (Wells, 1982 : xviii).

5. Les règles qui régissent ce type d'opposition mettent en jeu des critères morphologiques complexes brièvement décrits par Wells (1982 : 406).

6. Un parallèle éclairant peut être établi avec le fait que, dans l'accent «standard » britannique, les consonnes liquides / $/$ et $/ \mathrm{r} /$ sont dévoisées après une plosive sourde (surtout à l'initiale), ce qui constitue une forme d'aspiration.

7. En parallèle, Shuken (1984:156) mentionne aussi le voisement fréquent des consonnes phonologiquement sourdes. Ce phénomène semble avoir régressé puisqu'aucune occurrence de ce type ne figure dans nos données.

8. La rétroflexion des consonnes alvéolaires est également observée dans plusieurs variétés d'anglais américain, mais dans ces variétés d'outre-Atlantique, elle n'est pas aussi marquée, et ne se produit normalement qu'après $/ \mathrm{r} /$. (Cette précision est apportée suite à un échange avec Jacques Durand.)

9. Le même phénomène est observé dans certains dialectes d'anglais irlandais.

10. Il existe toutefois un certain degré d'incertitude quant à l'origine de ce schwa épenthétique, cette particularité étant aussi observée non seulement dans certaines variétés d'anglais irlandais (Wells, 1982 : 435), mais aussi dans le sud de l'Écosse (Durand, communication personnelle), zone géographique dans laquelle aucune influence celtique ne s'exerce.

11. Shuken (1984:157) note pour sa part que les variantes rétroflexes de /t/ et /d/ sont parfois observées indépendamment de $/ \mathrm{r}$ / et que la rétroflexion lui semblait constituer un ajustement articulatoire « fréquent, mais non systématique » (« common, but not consistent »).

12. Les données fournies consécutivement au dernier recensement, recensement organisé en 2011, sont difficilement comparables avec celles des recensements antérieurs car les documents disponibles ne précisent pas si la proportion de locuteurs de gaélique répond aux mêmes critères que ceux qui avaient été retenus auparavant. C'est ce qu'indique le point d'interrogation dans le tableau à côté de la proportion de locuteurs de gaélique pour l'année 2011.

13. Les environnements linguistiques dans lesquels se produit la réalisation glottale de $/ \mathrm{t} /$ forment un objet d'étude complexe (Wilhelm, $2011: 2-3$ ). Ils varient notamment en fonction des régions et de la classe sociale des locuteurs. Dans de nombreuses variétés d'anglais, ce type de réalisation de /t/ est, par ailleurs, attestée à l'initiale dans certains environnements, contrairement à l'idée reçue (cf. par exemple : See you tomorrow => ['si: jə ?ə'mDıəひ]). 
14. Voir cependant la section 2.2.5.

15. Les formants sont les fréquences les plus proéminentes des voyelles ou encore leurs harmoniques.

16. La présence d'UNBI en Irlande du Nord devrait alors être envisagée comme résultant des mouvements de population de l'Écosse vers l'Irlande du Nord lors des Plantations d'Ulster. Elle serait alors postérieure et non antérieure à sa présence en Écosse, contrairement à ce que suppose Cruttenden (1994).

\section{RÉSUMÉS}

La variété d'anglais parlée dans les Hébrides extérieures s'apparente, sur les plans grammatical et lexical, au Standard Scottish English (SSE). Sur les plan phonétique et phonologique, en revanche, elle diffère profondément de cette variété standard, en grande partie en raison de l'influence et de l'interférence du gaélique écossais.

À partir de deux corpus d'enregistrements de locuteurs des îles de Lewis et Harris et d'observations réalisées ces seize dernières années dans les Hébrides extérieures, cet article propose une description des traits segmentaux et suprasegmentaux les plus saillants de l'accent hébridéen traditionnel.

Un examen des caractéristiques du parler de certains adolescents de Lewis et Harris indique que plusieurs changements sont en cours dans les Hébrides extérieures, que ceux-ci résultent de la diffusion d'innovations phonétiques isolées ou de la disparition progressive des variantes les plus marquée, connue sous le terme de nivellement dialectal. Ces changements semblent favorisés à a fois par le déclin du gaélique et par l'attitude négative de certains jeunes hébridéens à l'égard des valeurs traditionnelles de la communauté insulaire. A partir de données recueillies auprès des adolescents et d'observations informelles, nous tentons d'identifier certains des traits les plus significatifs qui caractérisent l'évolution de l'accent de Lewis et Harris en temps apparent.

From a grammatical and lexical point of view, the variety of English spoken in the Outer Hebrides is akin to Standard Scottish English (SSE). From a phonetic and phonological point of view, however, it is absolutely distinctive, mainly because of the influence and interference of Scottish Gaelic.

Based on two corpora composed of recordings of speakers from Lewis and Harris as well as on informal observations collected in the Outer Hebrides during the past sixteen years, this paper identifies and describes the most salient traits of traditional Hebridean accents, be they segmental or prosodic.

A brief examination of the speech of Hebridean adolescents highlights several changes in progress. These are to be traced both from the diffusion of individual phonetic innovations and from the attrition of marked variants - a phenomenon known as dialect levelling. These changes seem to be facilitated both by the decline of the Gaelic language and by the rejection of the traditional Hebridean values by a number of young speakers. Using data collected from adolescents as well as informal observations, we try to identify some of the most prominent changes that affect the accents of Lewis and Harris in apparent time. 
INDEX

Keywords : Hebridean English, sociolinguistics, language variation and change, intonation, suprasegmentals, UNBI, voice quality, dialect levelling, geographical diffusion

Mots-clés : Anglais des îles Hébrides, sociolinguistique, variation, changement linguistique, intonation, éléments suprasegmentaux, UNBI, qualité de voix, nivellement dialectal, diffusion géographique

\section{AUTEUR}

\section{STEPHAN WILHELM}

Laboratoire TIL, Université de Bourgogne

wilhelmstephan@yahoo.com 